\title{
Electronic Correlations in Oligo-acene and -thiophene Organic Molecular Crystals
}

\author{
Geert Brocks ${ }^{1}$, Jeroen van den Brink ${ }^{1,2}$ and Alberto F. Morpurgo ${ }^{3}$ \\ ${ }^{1}$ Faculty of Science and Technology and MESA+ Research Institute, \\ University of Twente, P.O. Box 217, 7500 AE Enschede, The Netherlands \\ ${ }^{2}$ Institute-Lorentz for Theoretical Physics, Leiden University, \\ P.O. Box 9506, 2300 RA Leiden, The Netherlands \\ ${ }^{3}$ Kavli Institute of Nanoscience, Delft University of Technology, Delft, The Netherlands
}

(Dated: November 15, 2018)

\begin{abstract}
From first principles calculations we determine the Coulomb interaction between two holes on oligo-acene and -thiophene molecules in a crystal, as a function of the oligomer length. The electronic polarization of the molecules that surround the charged oligomer, reduces the bare Coulomb repulsion between the holes by approximately a factor of two. The effects of relaxing the molecular geometry in the presence of holes is found to be significantly smaller. In all cases the effective holehole repulsion is much larger than the valence bandwidth, which implies that at high doping levels the properties of these organic semiconductors are determined by electron-electron correlations.
\end{abstract}

PACS numbers: $71.20 . \mathrm{Rv}, 71.27 .+\mathrm{a}, 71.38 .-\mathrm{k}$

A unique aspect of organic molecular crystals is that the electronic properties of these solids bear the marks of both molecular and condensed matter physics. In a crystal the organic molecules preserve their identity, since molecular crystals are held together by van der Waals interactions without the formation of intermolecular covalent bonds. This results in a small overlap between the wave functions of electrons located on adjacent molecules, which leads to rather narrow electronic bands. Because of the small electronic bandwidths in these materials, the energy associated with interactions such as the electron-phonon or electron-electron interaction, may dominate over the kinetic energy of the charge carriers.

At a low density of charge carriers the interaction with phonons is expected to be dominant, which possibly leads to the formation of polarons 11. At increased carrier density -approaching one carrier per molecule- the role of electron-electron interactions becomes more and more important. In this regime Coulomb interactions may stabilize correlated magnetic ground states.

Probably the most studied example of organic conductors in the high-density regime is that of $\mathrm{C}_{60}$. In this material it has been shown experimentally that the Coulomb repulsion between two carriers on the same molecule is substantially larger than the electronic bandwidth [2]. At high carrier concentrations, the large Coulomb interaction results in a strongly correlated ground state. Both $\mathrm{K}_{4} \mathrm{C}_{60}$ and ammoniated $\mathrm{K}_{3} \mathrm{C}_{60}$ are Mott-Hubbard insulators, for instance $[3,4]$.

It can be expected that electron-electron interactions are not only important in $\mathrm{C}_{60}$ crystals, but also in crystals of many other molecules. In this paper we consider oligo-acenes and oligo-thiophenes, two classes of molecules often used in organic electronic applications, and we study their electronic structure in the high density regime using first principles calculations [5].

For all the molecules of experimental interest we find that the Coulomb interaction between two carriers on (a)
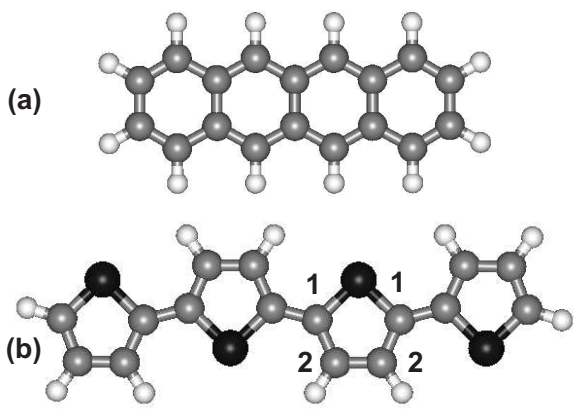

FIG. 1: Oligo-acenes are ladder-type hydrocarbons and oligo-thiophenes consist of a chain of thiophene rings. The molecules (a) tetracene and (b) quater-thiophene are shown, which have $n=4$ rings. They are planar and have (a) $D_{2 h}$ and (b) $C_{2 h}$ symmetry. The black, grey and white balls represent sulphur, carbon and hydrogen atoms.

the same molecule is much larger than the electronic bandwidth, also when the electronic screening of this interaction in the crystal is taken into account. Our results indicate that, analogous to $\mathrm{C}_{60}$, crystals of oligoacenes and -thiophenes should be viewed as correlated electron systems. In addition we find that, compared to the Coulomb interaction, the energy associated with relaxing the molecular geometry upon charging is smaller. This implies that the charge-charge Coulomb repulsion is the dominant factor at high carrier density. Correlation effects in these systems should be even more pronounced than in $\mathrm{C}_{60}$. Many of the electronic molecular levels in $\mathrm{C}_{60}$ are degenerate due to the high symmetry of this molecule and such degeneracies effectively reduce the Coulomb interaction [6]. In contrast, the symmetry of oligo-acene and -thiophene molecules (see Fig. 11) is much lower and all their molecular levels are non-degenerate.

Bare molecular Coulomb interactions. We first present the results obtained for the Coulomb interaction strength 
$U_{\text {bare }}$ between holes on single, isolated oligo-acene or thiophene molecules of different sizes. They are indicated by $n$, the number of (benzene or thiophene) rings comprising the oligomer, cf. Fig. 1] To establish the value of $U_{\text {bare }}$ we first consider two singly charged $n^{+}$ions far apart. The bare Coulomb interaction is then determined by the process in which a single electron is moved from one molecule to the other, i.e. $n^{+}+n^{+} \rightarrow n+n^{2+}$. Consequently the molecular Coulomb repulsion for two holes can be expressed in terms of the total energies of the neutral, doubly and singly charged molecules as $U_{\text {bare }}=E(n)+E\left(n^{2+}\right)-2 E\left(n^{+}\right)$.

Only ground state energies of individual molecules and ions enter here, which can be obtained with high accuracy from first-principles electronic structure calculations. The results presented here are obtained by density functional theory (DFT) in the local density approximation; using a generalized gradient functional gave only small differences [7]. Pseudo-potentials are used to represent the atomic cores [8] and the valence electronic wave functions are expanded in a plane wave basis set. Molecular calculations are performed by enclosing the molecule in a finite box [9], whereas the crystal calculations use periodic boundary conditions. This scheme has proved to yield accurate results for molecular crystals [10], polythiophene 11] and oligo-thiophene dimers [12].

The calculated bare Coulomb interaction $U_{\text {bare }}$ between two holes on a $n$-acene or $n$-thiophene molecule, $n$ ranging from 2 to 5 and from 4 to 16 , respectively, is listed in Table I, where the molecules are ordered according to increasing length. $U_{\text {bare }}$ ranges from 5.3 to $1.5 \mathrm{eV}$; it decreases as the length of the molecule increases. On conjugated molecules the holes are delocalized over the molecule, so their average distance is larger on a larger molecule, which of course lowers their Coulomb repulsion.

Molecular relaxations. So far we have not incorporated the fact that a charged molecule relaxes its geometry, which lowers its energy. This relaxation depends on the charge state of the molecule and effectively reduces the bare Coulomb interaction. The relaxation energy is the energy gained when optimizing the geometry of a charged molecule, starting from the geometry in its neutral state.

As for the bare Coulomb interactions, the relaxation energies also decrease with increasing molecular length $n$. Upon charging an oligo-thiophene molecule, it changes from an aromatic to a quinoid geometry [10]. A good measure for characterizing the change is to monitor the difference $\Delta r$ between the $\mathrm{C}_{1}-\mathrm{C}_{2}$ and $\mathrm{C}_{2}-\mathrm{C}_{2}$ bond lengths, cf. Fig. 1] where $\Delta r<0$ for the aromatic structure, and $\Delta r>0$ for the quinoid structure. The decrease of the relaxation energy $E_{\text {relax }}$ with increasing length $n$ can be understood qualitatively assuming that the excess charge is delocalized over the molecule. The induced change of $\Delta r$ should then roughly be proportional to the average excess charge density, i.e. $\Delta r \propto Z / n$, where $Z=1(Z=2)$ for the singly (doubly) charged molecule.

\begin{tabular}{c|cccc|ccccc}
\hline \hline & \multicolumn{4}{|c|}{$n$-acenes } & \multicolumn{5}{c}{$n$-thiophenes } \\
\hline$n$ & 2 & 3 & 4 & 5 & 4 & 6 & 8 & 12 & 16 \\
\hline$U_{\text {bare }}$ & 5.26 & 4.51 & 4.01 & 3.63 & 3.54 & 2.82 & 2.46 & 1.79 & 1.48 \\
$U_{\text {relax }}$ & 5.10 & 4.42 & 3.95 & 3.59 & 3.34 & 2.71 & 2.37 & 1.69 & 1.38 \\
$U_{\text {relax }}^{\text {AM1 }}$ & 5.00 & 4.32 & 3.86 & 3.52 & 3.05 & 2.25 & 1.85 & 1.79 & 1.48 \\
$U_{\text {eff }}^{\epsilon}$ & 2.47 & 1.99 & 1.75 & 1.59 & 1.47 & 1.21 & 1.09 & 0.70 & 0.56 \\
$E_{\text {pol }}^{+}$ & 1.22 & 1.23 & 1.15 & 1.10 & 0.95 & 0.80 & 0.69 & 0.52 & 0.44 \\
\hline$U_{\text {eff }}$ & 2.67 & 1.96 & 1.65 & 1.39 & 1.43 & 1.10 & 0.98 & 0.64 & 0.50 \\
$W$ & 0.35 & 0.34 & 0.39 & 0.61 & 0.36 & 0.34 & 0.31 & 0.30 & 0.30 \\
\hline \hline
\end{tabular}

TABLE I: Coulomb interaction $U$ between two holes on $n$ acene and $n$ - thiophene molecules. $U_{\text {bare }}$ denotes the bare interaction; $U_{\text {relax }}$ and $U_{\text {relax }}^{\text {AM1 }}$ denote the relaxed interaction calculated with DFT and AM1, respectively (see text, 15]); $U_{\text {eff }}^{\epsilon}$, and $U_{\text {eff }}$ denote effective interaction, calculated with the continuum model and the discrete dipole model, respectively. $E_{\text {pol }}^{+}$denote the polarization energies calculated from the discrete dipole model. The bottom row lists the valence bandwidths of the molecular crystals. All energies are in $\mathrm{eV}$.

Fig. [2] in which $\Delta r$ obtained from the optimized geometry is plotted against $Z / n$, demonstrates that the relationship is indeed approximately linear. In a harmonic approximation the relaxation energy per thiophene ring is proportional to $(\Delta r)^{2}$. For an oligomer consisting of $n$ rings, we then find $E_{\text {relax }}^{Z+}(n) \propto n(\Delta r)^{2} \propto Z^{2} / n$. The calculated relaxation energies indeed follow this scaling. Similar geometry changes take place upon charging the oligo-acene molecules, although the pattern is less uniform. Their relaxation energies are much smaller, but also follow the $E_{\text {relax }}^{Z+}(n) \propto Z^{2} / n$ behavior.

Yet the absolute value of the relaxation energies is small compared to the Coulomb interaction. The "relaxed" Coulomb interaction is defined as $U_{\text {relax }}=U_{\text {bare }}-$ $\left(E_{\text {relax }}^{2+}-2 E_{\text {relax }}^{+}\right)$. The inclusion of molecular relaxation upon charging changes the Coulomb interaction by less than $10 \%$; compare $U_{\text {bare }}$ and $U_{\text {relax }}$ in Table I.

Meanwhile, it has been argued that DFT calculations underestimate relaxation energies 13]. Therefore, we have also calculated the latter using the AM1 semiempirical Hartree-Fock method [14], which is known to yield a substantially larger relaxation and is likely to give an upperbound to the relaxation energies. The inclusion of AM1 instead of DFT relaxation energies leads to $U_{\text {relax }}^{\mathrm{AM} 1}$ in Table I. For oligo-thiophenes the AM1 relaxation effects are indeed much larger [13, 15], whereas for oligoacenes these effects are marginal. Note however that, irrespective of the technique used to calculate relaxations, the Coulomb interaction is dominant.

We therefore conclude that in the high density regime the nature of the ground state and low energy quasiparticle excitations is foremost determined by electronelectron interaction effects. The electron-phonon coupling is then a secondary effect which may result in a renormalization of quasi-particle properties. 


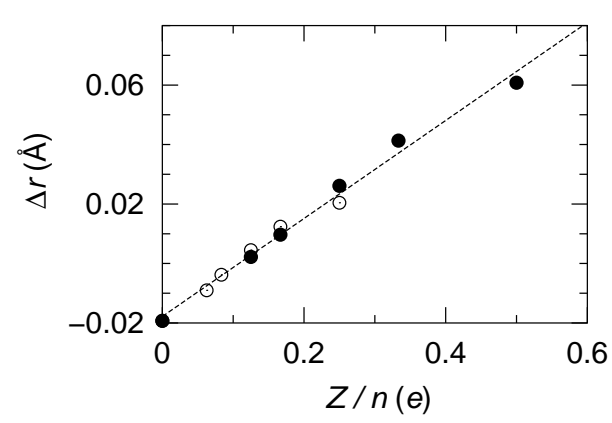

FIG. 2: The bond length change upon charging of $n$-thiophene molecules as function of the average excess charge density $Z / n$. The open and closed circles represent data from singly and doubly charged molecules; the line is a linear fit.

Screened Coulomb interactions. Having discussed the response of a single molecule to the creation of holes, we will now consider the response of the molecular crystal. In the presence of excess charges the electronic system of the crystal reacts to effectively screen the Coulomb interaction between the charges. The electronic screening due to electrons on the same molecule on which the charge(s) reside is already implicitly accounted for in the calculation of $U_{\text {bare }}$. We determine the screening energy due to all molecules that surround a charged molecule by calculating the polarization of these molecules in a discrete dipole model, see Refs. 16, 17. If we denote the total polarization energy that results from singly/doubly charged molecules by $E_{\text {pol }}^{+/ 2+}$, the effective screened Coulomb interaction is given by $U_{\text {eff }}=U_{\text {relax }}-\left(E_{\text {pol }}^{2+}-2 E_{\text {pol }}^{+}\right)$.

In order to evaluate the polarization energy $E_{\mathrm{pol}}^{+/ 2+}$, one needs to sum over the electric dipole moments on all molecules of the crystal lattice, induced by the excess charge [16, 17]. The strength of an induced dipole is proportional to the local electric field, which is the sum of the field due to the charge and of that due to all the other induced dipoles. The proportionality factor is the molecular polarizability tensor, whose values for oligo-acenes and -thiophenes are taken from Refs. 18 and 19. The lattice sum is determined by the crystal structures, which are taken from Refs. 20, 21, 22, 23. Large molecules are not well represented by point charges or point polarizability tensors. We therefore use the sub-molecular approach, where the charge or polarizability is distributed over the molecule by assigning a (sub-molecular) charge or polarizability to each benzene or thiophene ring $[1,18]$.

One complicating factor for 4- and 6-thiophene is that at room temperature two crystal structures exist, called the LT and HT phases 22. In practice the calculated $E_{\mathrm{pol}}^{+}$turns out to be the same within $0.01 \mathrm{eV}$ for these two phases, so we will not list them separately. Experimental crystal structures for 12- and 16-thiophene are not available and, for purpose of comparison only, we artificially construct their crystal structures by starting from the HT phase of 6-thiophene and enlarging the crystal axis that points along the long molecular axis, such as to accommodate the longer 12- or 16-thiophene molecule.

The results for the polarization energy $E_{\text {pol }}^{+}$are shown in Table I. The values are between 1.2 and $0.4 \mathrm{eV}$, where increasing the length $n$ of the molecule decreases the polarization energy. This is basically a volume effect; for longer molecules the charge is distributed over a larger volume, which "dilutes" its electric field. Whereas the polarizability per unit volume of the surrounding molecules increases somewhat with the size of the conjugated molecule, this dependence is weak and it does not compensate for the weaker average electric field.

The polarization energy is slightly different if one uses the ionic charge distribution given by the AM1 calculations. This is more localized than the DFT charge distribution, leading to a higher $E_{\mathrm{pol}}^{+}$. In practice we observe this only in the larger thiophenes, where $E_{\mathrm{pol}}^{+}$becomes 0.87, 0.79 and $0.72 \mathrm{eV}$ for 6 -, 8- and 12-thiophene.

At this point we are able to determine the effective Coulomb interaction $U_{\text {eff }}$ and the results are shown in Table I. Using AM1 charges, $U_{\text {eff }}$ changes by less than $0.06 \mathrm{eV}$ even for the larger thiophenes, so we have not listed these AM1 results. One observes that screening due to polarization of the surrounding molecules has an effect on the Coulomb interaction which is almost an order of magnitude larger than that of molecular relaxation. In fact, the screening is such, that the effective Coulomb interaction $U_{\text {eff }}$ is less than half $U_{\text {bare }}$.

Since polarization has such a large effect, one would like to establish the sensitivity of the results upon the model used to calculate the polarization energy. Therefore we have also calculated the polarization energies $E_{\text {pol }}^{+/ 2+}$ using a conceptually much simpler, but cruder continuum model. In such a model the charged molecule is placed inside a cavity surrounded by a homogeneous dielectric medium, where the cavity is shaped according to the molecule. The polarization energy is then given by the electrostatic interaction between the molecular charge distribution and the surrounding medium [12]. Table I also contains the results for the screened interaction $U_{\text {eff }}^{\varepsilon}$ based upon polarization energies calculated using a homogeneous dielectric medium. We have used a dielectric constant $\varepsilon=3$ for the oligo-thiophenes, as well as for naphthalene (2-acene) and $\varepsilon=3.3$ for the other oligoacenes [18]. One observes that the screened interaction calculated with the crude continuum model is roughly within $10 \%$ of the microscopic discrete dipole model. Moreover, $U_{\text {bare }}-U_{\text {eff }}^{\varepsilon}=E_{\text {pol }}^{2+}-2 E_{\text {pol }}^{+}$scales as $(\varepsilon-1) / \varepsilon$ so it is not very sensitive to moderate changes of $\varepsilon$ [12]. We conclude that the values of the screened Coulomb interaction obtained by the discrete dipole model are not very sensitive to the details of that model.

Coulomb interaction versus bandwidth. To determine the correlation strengths, the effective Coulomb inter- 


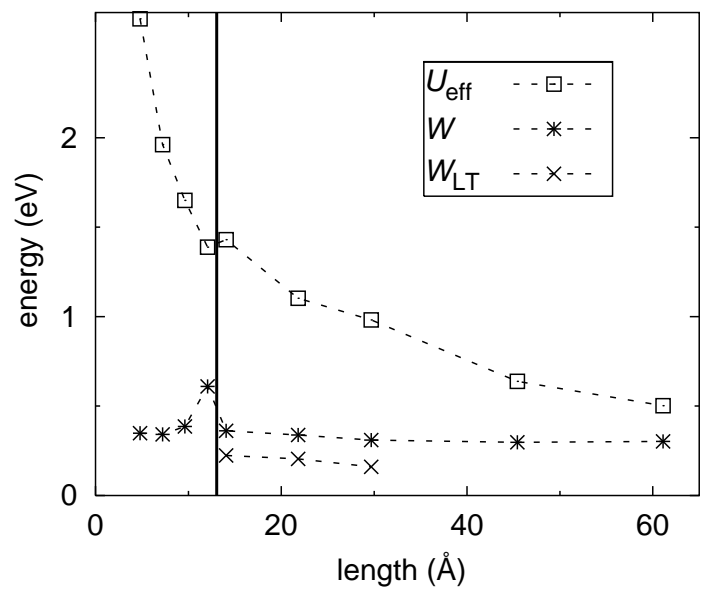

FIG. 3: The effective Coulomb interaction $U_{\text {eff }}$ and valence bandwidth $W$ ( $W_{\mathrm{LT}}$ for the LT phase) as function of the oligomer length (defined as the distance between the two extremal carbon atoms). The data for the oligo-acenes and thiophenes are plotted left and right of the solid vertical line.

action $U_{\text {eff }}$ for holes should be compared to the bandwidth $W$ of the highest valence band. The latter can be obtained from DFT calculations on oligo-acene and -thiophene crystals. Crystals of the HT and LT phases of 4- and 6-thiophene have been produced, whereas 8thiophene is only reported in the LT phase 22]. Again, for purpose of comparison only, the crystal structures for the HT phases of 8-, 12- and 16-thiophene are artificially constructed in the way discussed above. The calculated valence bandwidths $W$ are shown in Table I. Values in the same range have been reported on the basis of semi-empirical calculations [24, 25]. Generally, the bandwidths depend only weakly on the size of the oligomer. For instance, it decreases from $0.36 \mathrm{eV}$ to $0.30 \mathrm{eV}$ going from 4- to 16-thiophene in the HT phase. Such a weak dependence can be explained by the observation that the highest valence band is mostly derived from the highest occupied molecular orbital and the latter has the same character for all oligo-thiophenes. The bandwidth in the LT phase (not shown in Table I) decreases from $0.22 \mathrm{eV}$, 0.21 to $0.16 \mathrm{eV}$ for $4-, 6$ - and 8-thiophene, respectively. These values are smaller than those of the HT phase because of the different packing of the molecules in the two phases. The bandwidths of the oligo-acenes are similar to those in the oligo-thiophenes; only that of pentacene is substantially larger.

Fig. 3] summarizes the main result of our study. The figure shows the calculated effective Coulomb interaction $U_{\text {eff }}$ and the bandwidth $W$ for the oligo- acenes and - thiophenes, as a function of their molecular lengths. For longer molecules $U_{\text {eff }}$ becomes smaller, but does not drop below $W$ for any size of molecule. For the molecules that are of main experimental interest at present, i.e. 4-, 6thiophene and the acenes, the ratio $U_{\text {eff }} / W$ is between 2.3 and 7.6. So we conclude that in these systems the intramolecular Coulomb interaction is very large compared to the electronic bandwidth. We therefore expect that when oligo-acene and thiophene molecular crystals are highly doped, correlation effects will be very prominent, giving rise to magnetically ordered states.

[1] E.A. Silinsh and V. Ćápek, Organic Molecular Crystals, (AIP Press, Woodbury, New York, 1994).

[2] R.W. Lof et al., Phys. Rev. Lett. 68, 3924 (1992).

[3] M. Knupfer and J. Fink, Phys. Rev. Lett. 79, 2714 (1997).

[4] T. Takenobu et al., Phys. Rev. Lett. 85, 381 (2000).

[5] The charge on the molecules is to be compensated by counter-ions, as in alkali-doped $\mathrm{C}_{60}$.

[6] O. Gunnarsson, Rev. Mod. Phys. 69, 575 (1997).

[7] J. Perdew and A. Zunger, Phys. Rev. B 23, 5048 (1981); J.P. Perdew, K. Burke, and M. Ernzerhof, Phys. Rev. Lett. 77, 3865 (1996).

[8] N. Troullier and J.L. Martins, Phys. Rev. B 43, 1993 (1991).

[9] R.N. Barnett and U. Landman, Phys. Rev. B 48, 2081 (1993).

[10] G. Brocks, Phys. Rev. B 55, 6816 (1997).

[11] G. Brocks, Theor. Chem. Acc. 104, 116 (2000).

[12] G. Brocks, J. Chem. Phys. 112, 5353 (2000).

[13] L. Zuppiroli et al., Chem. Phys. Lett. 374, 7 (2003).

[14] M. J. S. Dewar et al., J. Am. Chem. Soc. 107, 3902 (1985).

[15] For the largest oligo-thiophenes $E_{\text {relax }}^{2+}(n)=2 E_{\text {relax }}^{+}(n)$, see A. J. W. Tol, Chem. Phys. 208, 73 (1996).

[16] J. van den Brink et al., Phys. Rev. Lett. 75, 4658 (1995), ibid. 76, 2826 (1996).

[17] J. van den Brink and G.A. Sawatzky, Europhysics Letters 50, 447 (2000).

[18] I. Eisenstein and R.W. Munn, Chem. Phys. 77, 47 (1983).

[19] B. Champagne, D.H. Mosley and J.-M. André, J. Chem. Phys. 100, 2034 (1994).

[20] D. Holmes et al., Chem. Eur. J. 5, 3399 (1999).

[21] C.C. Mattheus et al., Acta Cryst. C 57, 939 (2001).

[22] D. Fichou, J. Mater. Chem. 10, 571 (2000).

[23] R.W.G. Wyckoff, Crystal Structures, (Interscience, New York, 1971), Vol. 6, part 2.

[24] R.C. Haddon et al., J. Mater. Chem. 5, 1719 (1995).

[25] J. Cornil et al., Adv. Mater. 12, 978 (2000). 